\title{
PLANTAS PALUSTRES E AQUÁTICAS QUE SE COMPORTAM COMO INVASO- RAS, NO ESTADO DE MINAS GERAIS
}

\author{
Mitzi Brandão1 \\ Júlio Pedro Laca-Buendia 2 \\ Manuel Losada Gavilanes²
}

\begin{abstract}
RESUMO - Foram cadastradas as espécies palustres e aquáticas com comportamento de plantas invasoras, quando da obstrução de canais de irrigação e drenagem de lagos, represas, ou ainda como competidoras em culturas irrigadas. As plantas cadastradas no presente trabalho somam um total de 113 espécies, englobando 64 gêneros e 40 famílias.
\end{abstract}

Palavras-chave: Plantas daninhas; Plantas aquáticas e palustres; Daninhas aquáticas.

ABSTRACT - Are listed the boggy and aquatic plants that behave as weeds in the obstruction of drainage and irrigation canals, lakes, dams or irrigated crops, totalling 113 species, involving 64 genera and 40 families.

Key words: Weeds; Boggy and Aquatic planta; Aquatic weeds.

\section{Introdução}

No presente trabalho foram consideradas aquelas plantas que se comportavam como invasoras, nas culturas de arroz irrigado, como também mostravam-se problemáticas quando da conservação de canais (de irrigação, de drenagem, etc.) ou infestavam lagoas, represas e mananciais.

As espécies apresentadas estão entre as consideradas como plantas daninhas específicas dessas áreas, conforme Weldon et al. (1969), Vasconcellos (1970), Bristow et al. (1979), Riemer (1984) e Pereira \& Brandão (1988).

Embora consideradas problemáticas sob diversos aspectos, de acordo com o Silva \& Silva (1956) Bristow et al. (1979) e Weldon et al. (1969), é na cultura do arroz irrigado que essas plantas causam um maior impacto em termos de produção e competição, como atestam os trabalhos de Aranha et al. (1980, 1981) Sagastegui-Alva (1974), Rochelle et al. (1976) e Pereira \& Brandão (1988).

\section{Material e métodos}

Durante a realização de diversos projetos de campo ligados a EPAMIG, entre os anos 1975-1987, essas plantas foram coletadas em vários pontos do estado de Minas Gerais, a saber: represas-Três Marias, Marimbondo, Furnas, Águas Vermelhas, Volta Grande, São Simão, Itumbiara e Emborcação, lagoas-Lagoa Santa, Lagoa dos Mares, Lagoa da Pampulha, Lagoa dos peixes, Lagoa Feia, entre outras.

Farto material enviado por pesquisadores que trabalham com lavouras de arroz irrigado, situadas ao longo dos rios São Francisco, Gorutuba, Verde, Sapucaí, Grande, Paracatu, Paranaíba, constituiu-se em outra fonte de informações.

\footnotetext{
${ }^{1}$ Pesquisador EPAMIG, Caixa Postal 515, 30.188 - Belo Horizonte, MG. Bolsista CNPq.

2Pesquisador EPAMIG, Caixa Postal 515, 30.188 - Belo Horizonte, MG.

3 Professor de Botânica da ESAL, Caixa Postal 37, 37.200 - Lavras, MG. Bolsista do CNPq.
} 
As plantas coletadas foram identificadas de acordo com as normas clássicas da taxonomia botânica. Os termos utilizados para a caracterização dos habitats "flutuantes", "emersas" e "submersas" para aquelas de hábito aquático, podem ser assim definidos, segundo Ferreira (1975), em sua primeira edição do dicionário Aurélio: Flutuante, vem do latim "fructuante" = conservar-se à superficie de um líquido; termo que correlacionamos com as plantas que sobrenadam, de raízes livres como o aguapé. Emersas, do latim "emergere" = sair de onde estava mergulhado, termo que utilizamos para designar aquelas plantas aquáticas, que se encontram presas por suas raizes aos barrancos ou leito dos rios, mas tendo suas folhas, flores e frutos aparecendo acima da superfície das águas.

Submersas - do latim "submergere" = ficar inteiramente mergulhado na água, termo que escolhemos para denominar as plantas que se acham presas ao fundo, pelas suas raízes, mas cujas folhas e demais estruturas não afloram à superficie.

A palavra palustre, por sua vez, vem do latim "palustre" = pantanoso, alagadiço, termo esse que utilizamos para designar plantas que vivem na faixa de transição, entre a água livre e a terra firme.

Para a identificação das espécies, baseamo-nos̀ em dados colhidos em autores como: Seubert (1871), Urban (1879), Pio Correa (1926-1975), Hoehne (1948), Rudd (1955), Castelhanos (1959), Vasconcellos (1970), Bristow et al. (1979), entre outros.

Após terem sido identificadas, registradas, montadas e rotuladas, essas plantas foram para o acervo do PAMG/EPAMIG.

Essas espécies são agora listadas em ordem alfabética, segundo as famílias a que pertencem, seguidas de seu(s) nome(s) popular(es), região de coleta, posição em seus habitats, assim como o seu número no PAMG.

\section{Resultados}

Dando seqüência ao cadastramento das espécies invasoras de culturas no Estado de Minás Gerais, iniciado em 1973, os autores apresentam aqui aqueles que ocorrem nas lavouras de arroz irrigado e que infestam e bloqueiam canais de irrigação e drenagem, assim como lagoas e represas.

No Anexo 1, são relacionadas as espécies por ordem alfabética das famílias a que pertencem, seguidas de seu (s) nome (s) popular (es).

Neste mesmo Anexo, relacionam-se ainda as espécies, conforme os seus habitats (aquáticas e palustres) e sua distribuição nas regiões do Estado (Centro, Sul, Norte, Leste, Noroeste e Triângulo Mineiro).

O númerò total das espécies ascende a 113, contendo 66 gêneros englobados em 40 famílias. São referidas 73 espécies para o Triângulo Mineiro, 46 para o Noroeste do Estado, 60 para o Leste, 41 para o Norte, 83 para o Centro e 89 para o St...

\section{Discussão}

Dependendo da rapidez de instalação e multiplicação, certas espécies pertencentes às famílias Pontederiaceae, como Eichornia crassipes (Mart.) Solms. e Eichornia azurea Kunth., Heteranthera reniformis Ruiz et Pav. e Heteranthera limosa; Araceae: Pistia stratiotes L. e, entre as Salviniaceae: Salvinia auriculata Aubl. e Salvinia rotundifolia Willd., são de difícil controle, transformando-se efetivamente em invasoras altamente competitivas. O seu cadastramento e inclusão na listagem das plantas daninhas ocorrentes no Estado de Minas Gerais, iniciada por pesquisadores da EPAMIG em 1973, é uma decorrência desse comportamento. 
Outras espećies pertencentes aos gêneros Echinodorus, Sagittaria, Ceratophylum, Nymphoides, Myriophyllum, Egeria, Lemna, Spirodela, Utricularia, Mayaca, Nymphaea, Pontederia, Potamogeton, embora bloqueiem canais de irrigação e drenagem, não se comportam tão agressivamente.

Naquelas de hábito palustre, as pertencentes aos gêneros Alternanthera, Polygonum, Ludwigia, Spilanthes, Fimbrystilis, Rhynchospora, Scleria, Echinochloa, Typha, Paspalum, Hedychium, que formam populações densas, têm papel relevante na obstrução de canais de drenagem ou irrigação, margens de lagoas e represas, como também invadem e competem nas lavouras de arroz irrigado.

No gênero Cyperus certas espécies são sérias concorrentes em termos de nutrientes nas lavouras onde ocorrem, como o Cyperus ferax, Cyperus aciculares, Cyperus esculentus e Cyperus iria.

No gênero Paspalum, as espécies Paspalum repens, Paspalum conspersum, Paspalum acuminatum e Paspalum rivulare, chegam a bloquear os canais onde ocorrem.

Laersia hexandra, Echinochloa crus-pavonis e Oryza sativa (arroz vermelho) comportam-se da mesma forma que as espécies anteriormente citadas.

As demais espécies relacionadas, comportam-se também como invasoras, mas não se mostram tão problemáticas.

\section{Referências Bibliográficas}

ARANHA, C; LEITÃO fo, H.F. \& PIO, R.M. 1980. Plantas invasoras de várzeas no Estado de São Paulo. Plant. Daninha, Rio de Janeiro. 3 (2): 85-95.

ARANHA, C. \& PIO, R.M. 1981. Plantas invasoras de culturas de arroz (Oryza sativa L) no Estado de São Paulo, 1. Dicotiledôneas. Plant. Daninha. Rio de Janeiro 4 (1): $35-58$.

BEYRUTH, Z. 1981. Aproveitamento do Aguapé. In: TÓPICOS DE BOTÂNICA ECONÔMICA. São Paulo. 10p.

BRISTOW, J.M.; CARDENAS, J.; FULLESTON, T.M. \& SIERRA, J. 1979. Malezas aquáticas (aquatic weeds). Bogotá, Italgraf. 116p.

CASTELlanOS, A. 1959. Las Pontederidaceae do Brasil. Arq. Jard. Bot., Rio de Janeiro. 16: 149-216.

CORRÊA, M. Pio. 1926-1975. Dicionário das Plantas úteis do Brasil e das exóticas cultivadas. Rio de Janeiro, Imprensa Nacional, 6v.

HOEHNE, F.C. 1948. Plantas aquáticas. São Paulo, Imprensa Nacional. 168p. (Reimpressão).

MARTINS, H.F. \& CARAUTA, J.P.P. 1984. Plantas aquáticas-classificação e comentários. Atas Soc. Bot. Bras., Rio de Janeiro, 2 (13): 101-4.

OLIVEIRA, E. de C. 1980. Cyperaceae juss-morfologia dos aquênios dos gêneros ocorrentes no Brasil Rodriguésia. Rio de Janeiro, 32 (55): 327-405.

PEREIRA, R.P. \& BRANDÃO, M. 1988. Levantamento e identificação das plantas invasoras da cultura de arroz na região norte do Estado do Rio de Janeiro. Bol. Tec. Empresa Pesqui. Agropecu. Estado Rio J., Niterói, n? 12

RATAJ, K. 1978. Alismataceae no Brasil. Acta Amazon, Manaus, 8 (1): 1-53, Mar,

RIEMER, D.N. 1984. Introdution to freshwater vegetation, Westport. 207p.

ROCHELLE, L.A., BANZATTO, N.V. \& DELISTOIANOV, J. 1976. Levantamento botânico das plantas invasoras em culturas de arroz (Oryza sativa L.) irrigada. Rev. Agric., Piracicaba. 51 (3/4): 145-8

RUDD, X.E. 1955. The American species of Aeschynomene. Bull. U.S. natn. Mus., Washington. $173 \mathrm{p}$.

SAGASTEGUI ALVA, A. 1973. Manual de las malezas de la Costa Norperuana. Universidad National de Trujillo, 480p., il. 
SEUBERT, M. 1842-1871. Alismataceae, Pontederiaceae in: MARTIUS, K.F.P. VON; EICHLER, A.G. \& URBAN, L. Flora Brasiliensis. 1.

SILVA, G.M. \& SILVA, S.O. 1956. Ervas Daninhas em piscicultura. SEMINÁRIO DE HERBICIDAS E ERVAS DANINHAS, 1. p. 171-84.

URBAN, L. 1861-1879. Umbelliferae. In: MARTIUS, K.F.P. VON; EICHLER, A.G.\& URBAN, L. Flora Brasiliensis. 11, 1.

VASCONCELLOS, J. de C. 1970. Plantas (angiospérmicas) aquáticas, anfibias e ribeirinhas. Secretaria do Estado da Agricultura. Direção Geral dos Serviços Florestais e Agrícolas. Estudos e Divulgação Técnica. Lisboa. 253p.

WELDON, L.W.; BLACKBURN, R.D. \& HARRISON, D.S. Common aquatic weeds. 


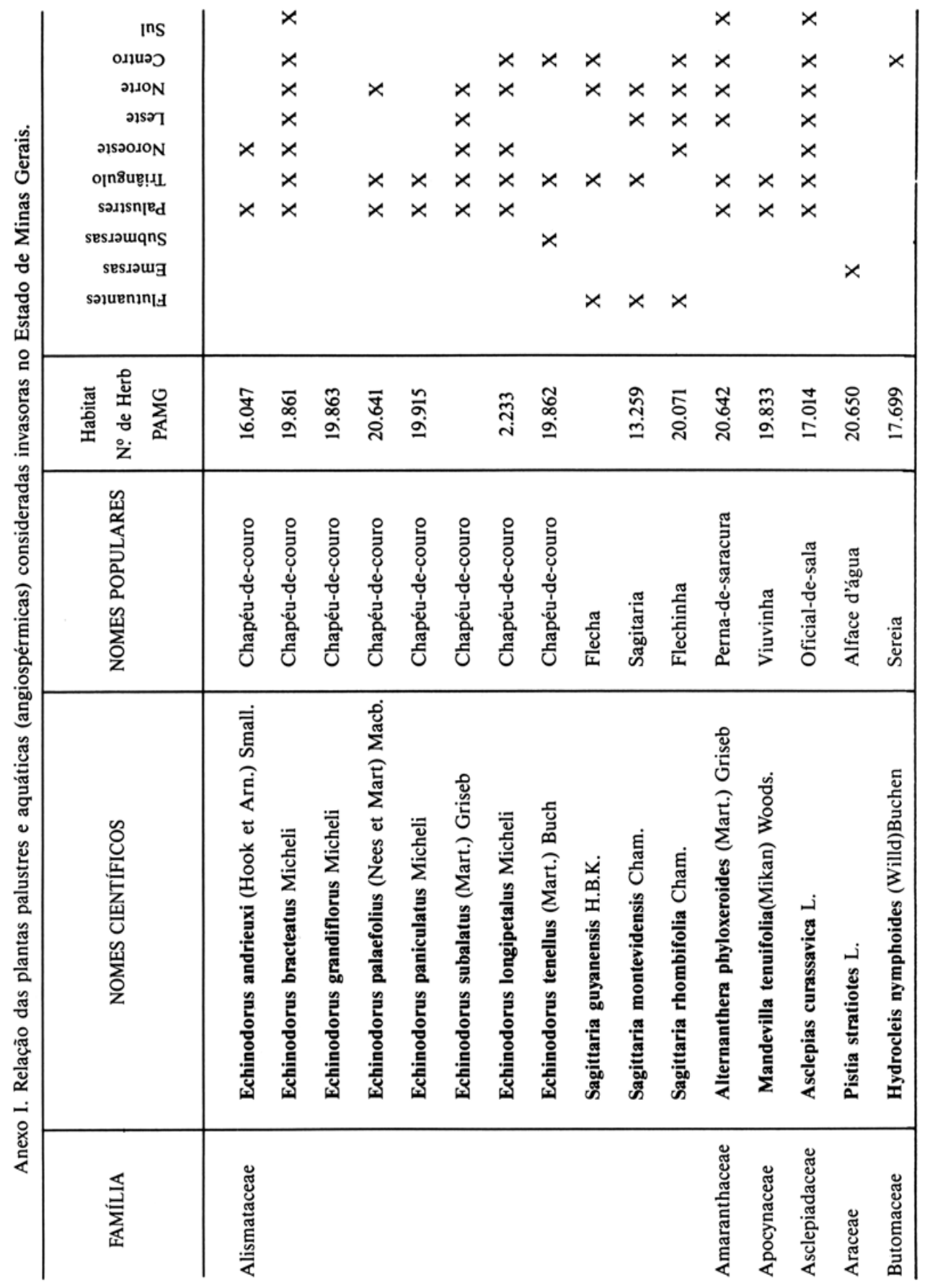




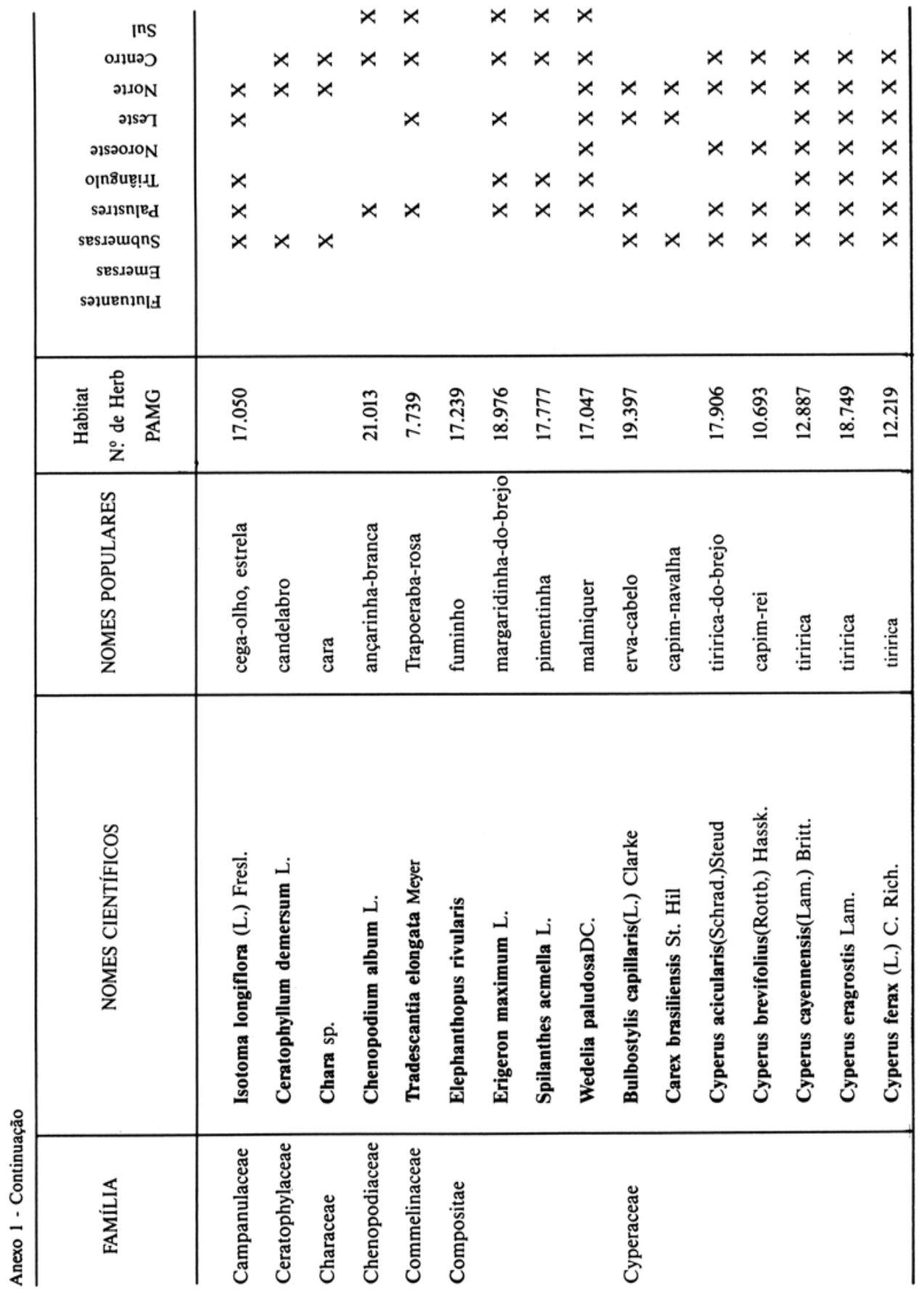




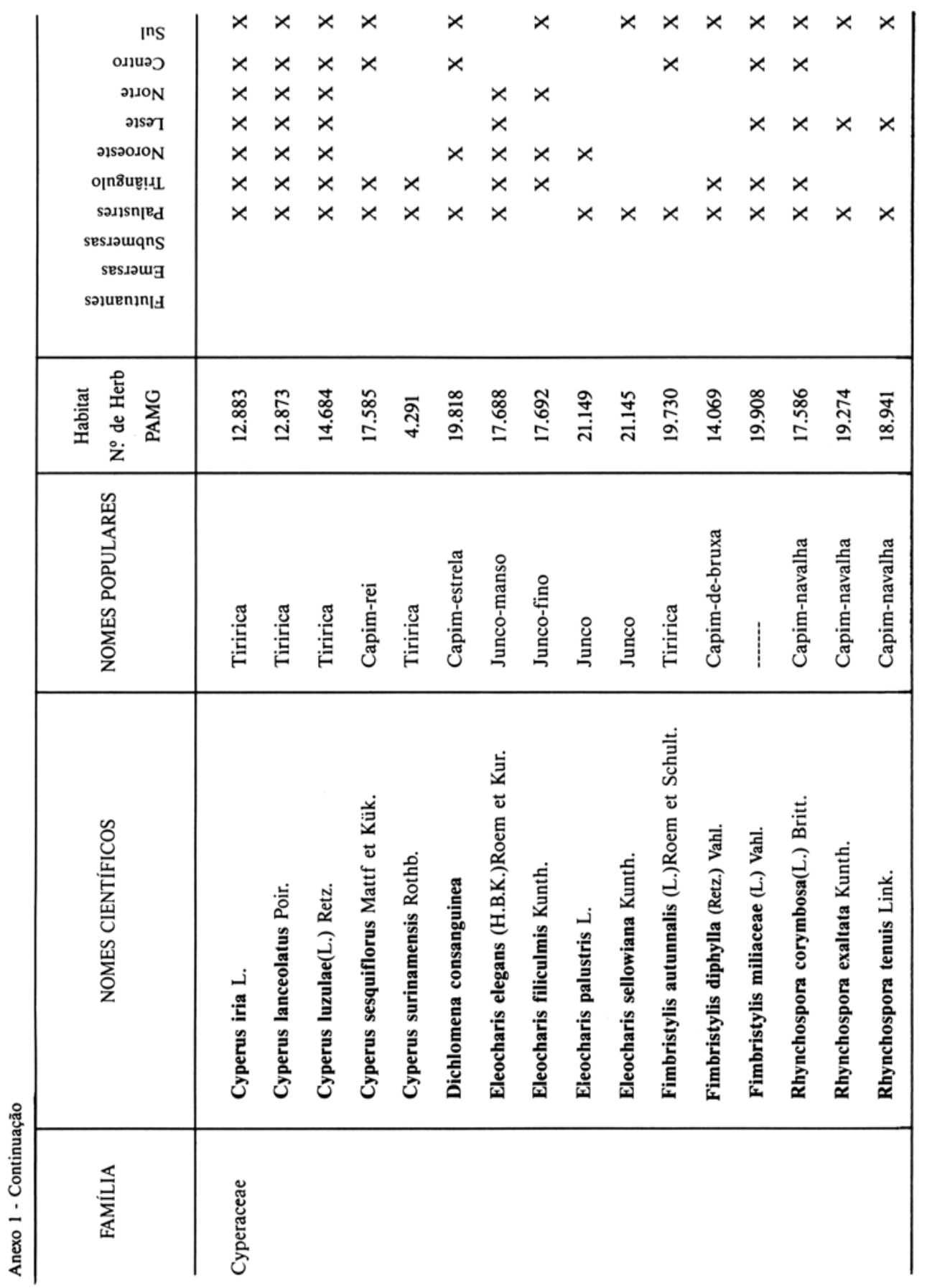




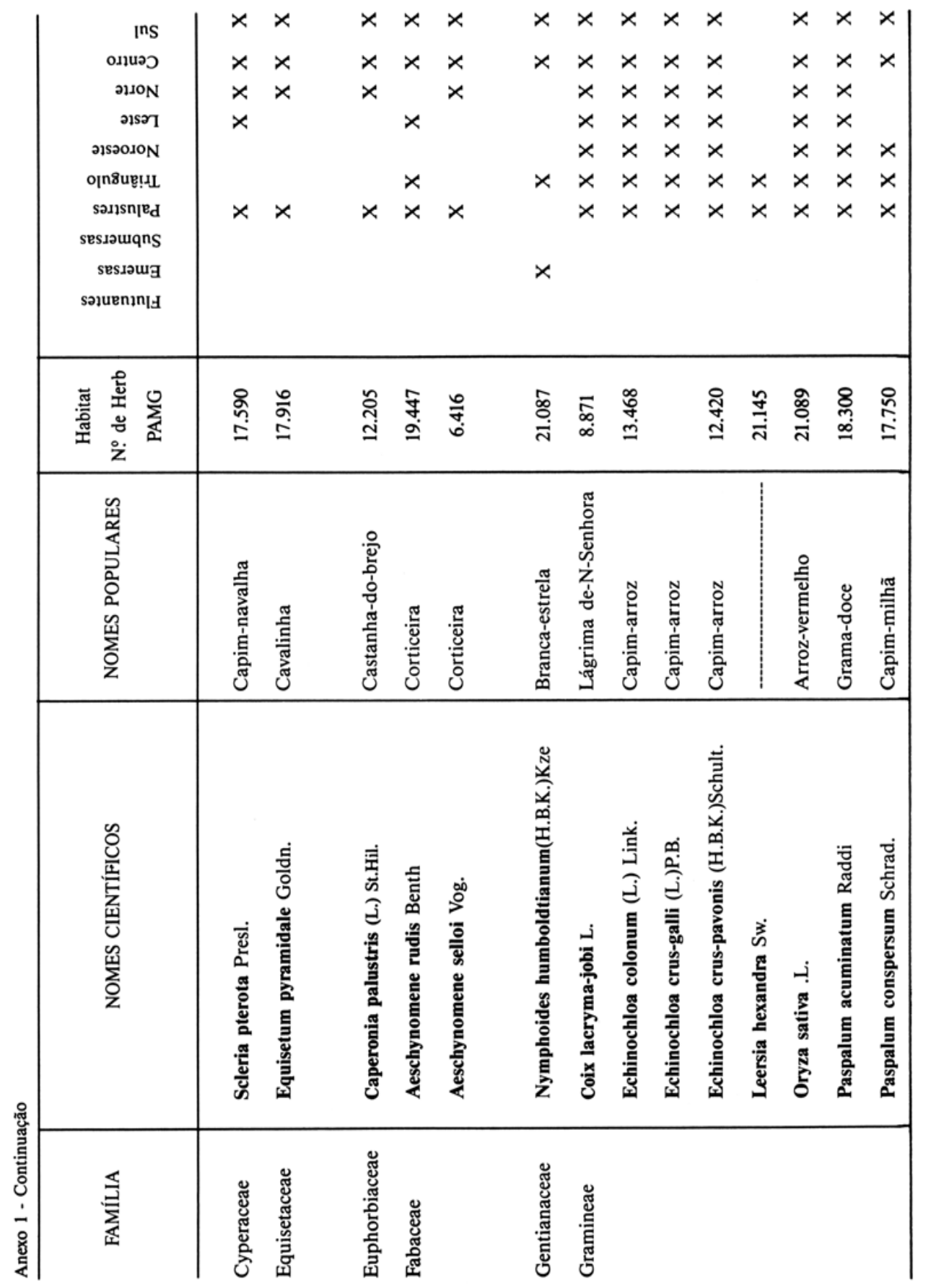




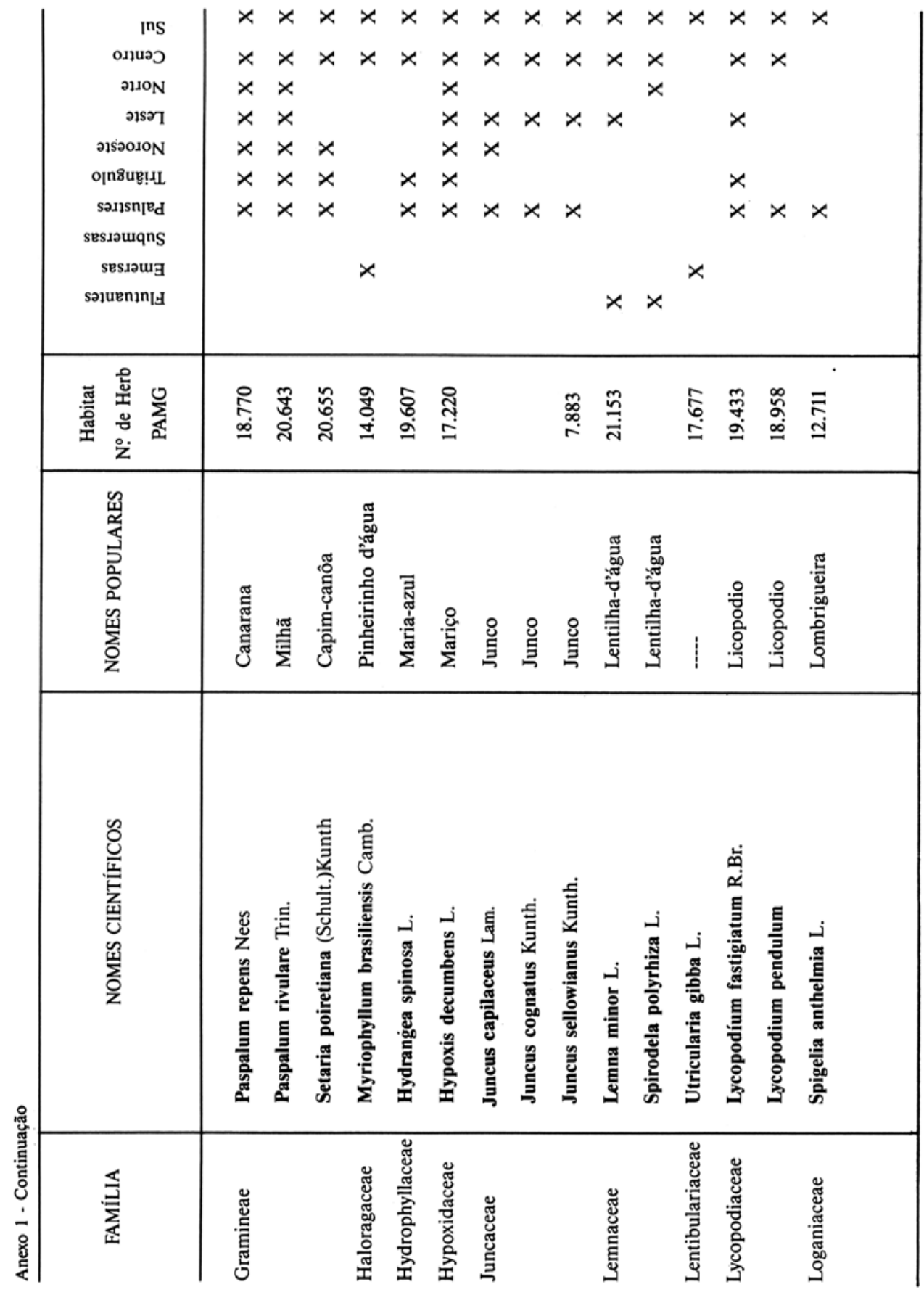




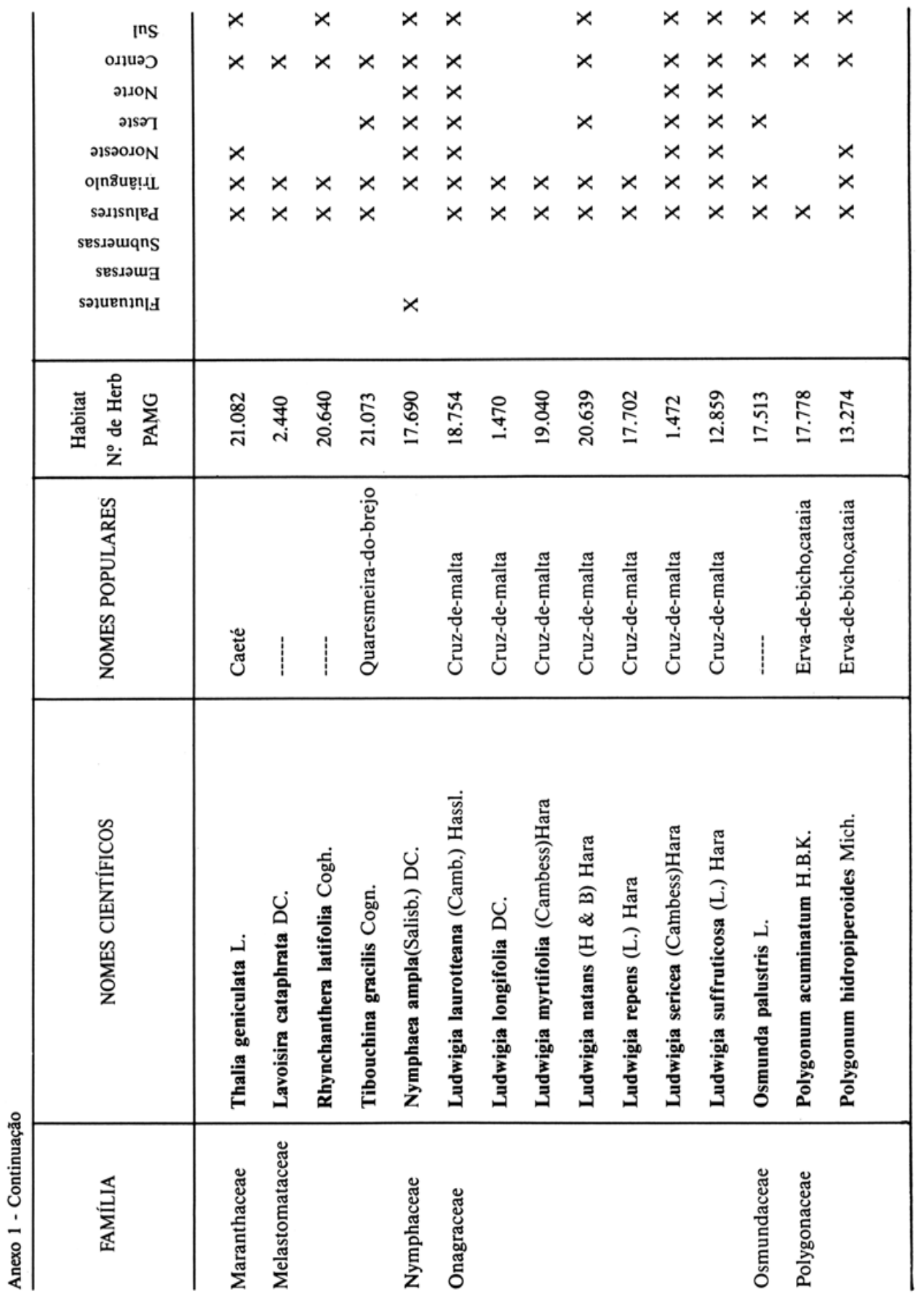




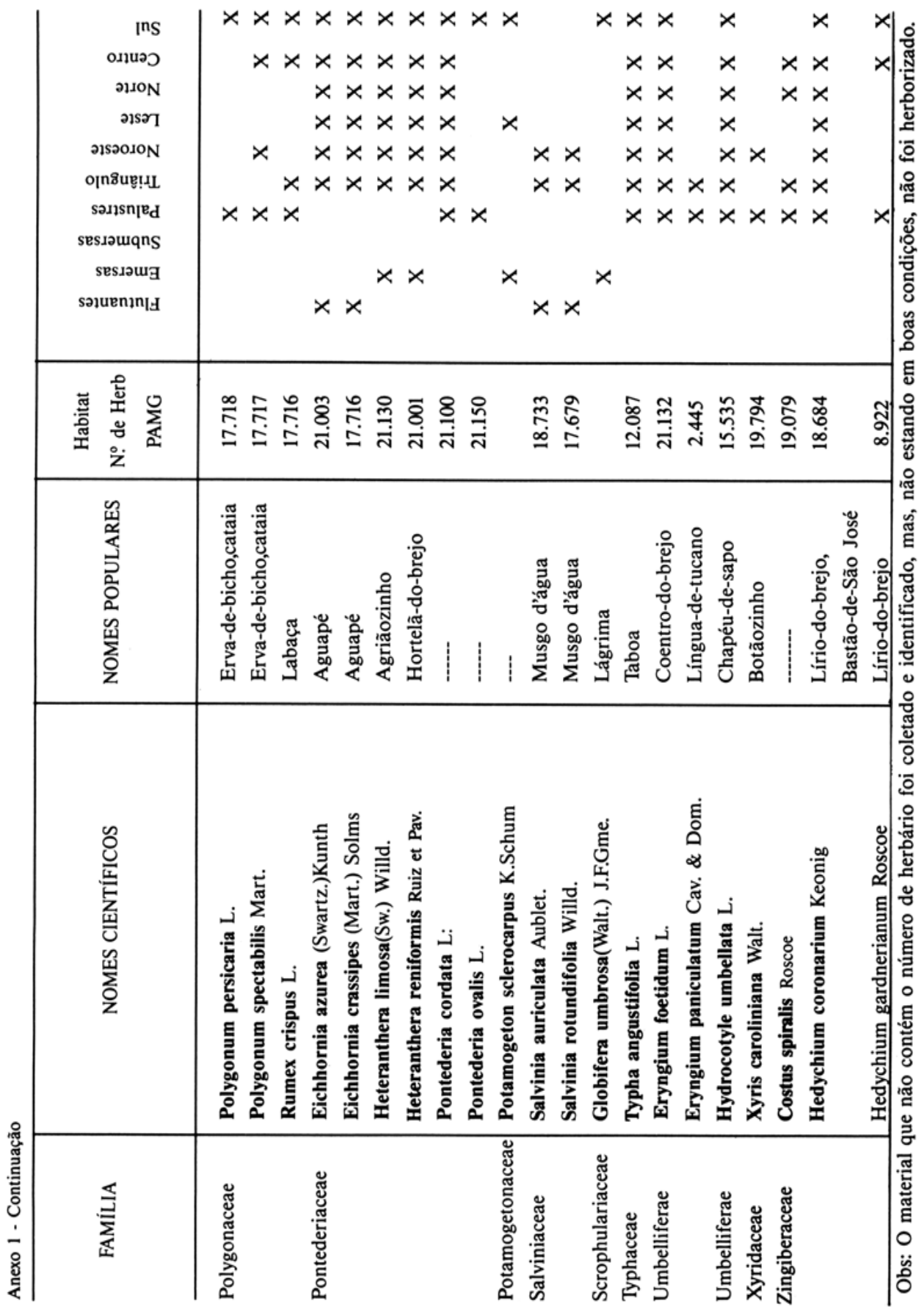

